\title{
Does the Type of Surgery After Preoperative Systemic Therapy for T3/T4 Breast Cancer Impact Survival?
}

\section{Jamila Alazhri ${ }^{1,4 *}$, Tulay Koru-Sengul ${ }^{2,3}$, Feng Miao $^{3}$, Margaret M Byrne $^{2,5}$ and Eli Avisar ${ }^{1,3}$}

${ }^{1}$ Division of Surgical Oncology, Department of Surgery, University of Miami Miller School of Medicine, Miami, FL, USA

${ }^{2}$ Department of Public Health Sciences, University of Miami Miller School of Medicine, Miami, FL, USA

${ }^{3}$ Sylvester Comprehensive Cancer Center, University of Miami Miller School of Medicine, Miami, FL, USA

${ }^{4}$ Division of Breast and Endocrine Surgery, Department of Surgery, King Fahad Specialist Hospital, Dammam, Saudi Arabia

${ }^{5}$ H. Lee Moffitt Cancer Center and Research Institute, Tampa, FL, USA

*Corresponding Author: Jamila Alazhri, Breast and Endocrine Surgery Division, Department of Surgery, King Fahad Specialist Hospital, Dammam, Saudi Arabia.
Received: April 01, 2020

Published: May 22, 2021

(c) All rights are reserved by Jamila

Alazhri., et al.

\begin{abstract}
Introduction: Our purpose was to study the impact of the extent of surgery on survival of women with T3/T4 breast cancer treated with preoperative systemic therapy (PST) in form of chemotherapy, hormonal therapy, or both.

Methods: Population-based Florida Cancer Data Registry was screened for women diagnosed with T3/T4 breast cancer who received PST followed by either breast conserving surgery (BCS) or mastectomy. A multivariable Cox regression model was used to identify significant predictors of overall survival. Adjusted hazard ratio (aHR) and 95\% confidence interval (95\%CI) were calculated.

Results: Out of 712 patients receiving PST, $72(10 \%)$ had BCS and 640 (90\%) had mastectomy. After covariable adjustment, patients who received both chemo and hormonal PST had better prognosis than patients with chemotherapy alone (aHR=1.90, 95\%CI: 1.20 - $3.01, \mathrm{p}=0.006)$ or hormonal therapy alone $(1.95,0.84-4.56, \mathrm{p}=0.122)$. Hispanic origin $(0.50,0.27-0.92, \mathrm{p}=0.027)$ positively impacted survival. Medicare $(1.70,1.08-2.68, p=0.021)$ and uninsured $(1.69,1.10-2.60, p=0.016)$ compared to private insurance, poorly-differentiated/undifferentiated $(2.24,1.04-4.82, \mathrm{p}=0.039)$ compared to well-differentiated grade, and distant SEER stage $(4.13,1.68-10.12, p=0.002)$ compared to localized were significant predictors of worse survival. There was no significant difference in survival between women who had mastectomy compared to BCS $(1.35,0.82-2.21, p=0.234)$. In the subgroup of patients who had mastectomy, radiotherapy resulted in significantly better survival than no radiotherapy (HR 0.68, 0.47-0.99, p = 0.044).

Conclusion: The type of surgery after PST for T3/T4 breast cancer does not impact overall survival. BCS could be considered for patients with T3/T4 tumors after PST.

Keywords: Locally Advanced Breast Cancer; T3 Breast Cancer; T4 Breast Cancer; Preoperative Systemic Therapy; Neoadjuvant Chemotherapy; Breast Conserving Surgery; Mastectomy; Florida
\end{abstract}

\section{Introduction}

Our previous work has suggested that Hispanic ethnicity, advanced SEER stage, and combined PST (Chemo and hormonal therapy) are significant predictors of receiving mastectomy for patients with T3/T4 breast cancer [1]. Weather the decision for mastectomy, as opposite to Breast conserving surgery (BCS), has impacted the patients' overall survival is an important question to answer. BCS is a safe alternative to mastectomy for the local treatment of breast cancer [2]. Similarly, breast conservation after preoperative systemic therapy (PST) was comparable to mastec- tomy in a variety of studies provided the appropriate selection of patients with a good response and obtaining negative pathologic margins [3]. In most studies, however, T3/T4 tumors are underrepresented raising the question of the safety of breast conservation in those patients.

We sought to study the overall survival in a large prospectively collected population-based cancer incidence database of T3/T4 breast cancer patients treated with PST, followed by either BCS or mastectomy. 


\section{Materials and Methods}

\section{Study population}

Population-based Florida Cancer Data System (FCDS) registry was screened for women diagnosed with T3/T4 breast cancer from 1996 to 2007 who received PST (chemotherapy only or hormonal therapy only or both) followed by either BCS or mastectomy. Florida's Agency for Health Care Administration (AHCA) database provided procedure and diagnoses information from all in- and out-patient facilities, and data from the US census provided a proxy for individual level by neighborhood level socioeconomic status (SES). All three sources of data (FCDS, AHCA, US Census) were linked for female T3/T4 breast cancer patients who were 18 years or older and resided in Florida during the study period at the time of their primary breast cancer diagnosis. Patients with carcinoma in situ or with missing data on age, race, ethnicity, SES, type of surgery, survival were excluded from the study.

\section{Variables}

Overall survival, the primary outcome variable, was defined as the elapsed time from primary breast cancer diagnosis during 1997-2007 to death or last encounter for alive patients; the patients were followed for an additional three years period, until 2010 , to better evaluate survival. Therefore, all women included in this study had at least three years of follow-up. The type of surgical treatment was classified as whether the patient had either BCS or mastectomy. Patients' sociodemographic variables were age at diagnosis (years), race (White, Black, Other), ethnicity (Hispanic and non-Hispanic), primary payer at diagnosis (private insurance, Medicare, Medicaid, defense/military, Indian Health Service, other insurance, or uninsured). Patients' SES was determined based on the patients' neighborhood i.e. census track at the time of the diagnosis as the percent of individuals living below the federal poverty line from US census tract-level information with four categories as lowest ( $\geq 20 \%$ ), middle-low ( $\geq 10 \%$ and $<20 \%$ ), middle-high ( $\geq 5 \%$ and $<10 \%$ ), and highest $(<5 \%$ ). Clinical characteristics included tumor related variables such as SEER stage, histological type and differentiation grade, and patients' co-morbidities that was determined by the 31-categoryElixhauser Comorbidity Index and were reported as an aggregated variable to reflect the number of comorbidities as $0,1-2,3-4$ and $>4$.

\section{Statistical analysis}

Sociodemographic and clinical characteristics of patients were presented as frequencies and percentages for categorical variables and means and standard deviations (Sd) for continuous variables, and compared for all patients in the study, and by type of surgery (BCS or mastectomy). Kaplan-Meier method and log-rank test were used to compare survival time between several groups such as types of surgery, PST, and radiation therapy. A multivariate Cox regression model was used to calculate adjusted hazard ratios (aHR) and corresponding 95\% confidence intervals (95\% $\mathrm{CI}$ ) along with p-values by including sociodemographic, tumor and treatment related covariates and number of comorbidities. Statis- tical significance was considered if $\mathrm{p}$-value is $<0.05$ i.e. $5 \%$ type-I error rate. All statistical analyses were performed using SAS v9.4 for Windows (SAS Institute Inc., Cary, NC, USA). The study was approved by the Institutional Review Boards of both University of Miami Miller School of Medicine and Florida Department of Health.

\section{Results}

Inclusion and exclusion criteria were satisfied by 712 patients. Out of the total number of 712 analyzed patients $72(10 \%)$ had BCS and $640(90 \%)$ had mastectomy after PST. The socio-demographic characteristics of the sample are shown in table 1 . The median follow up was 29.4 months, ranging between 0.5 - 133.1 months. By the time of last follow-up, $73.6 \%$ of the patients who received BCS and $67.7 \%$ of the patients who received mastectomy were alive. The mean age was comparable between BCS group (mean 55.5 with range 29 - 90 years) and the mastectomy group (55.4; 22 95). Most of the patients were White (76.7\%); about $68 \%$ of BCS patients and $77.7 \%$ of mastectomy, and non-Hispanic; $93.1 \%$ of the BCS group, and $86.3 \%$ of the mastectomy group. The percentage distribution of the SES, represented by the neighborhood poverty in the BCS and mastectomy groups was $18.1 \%$ versus $18.4 \%$ for lowest, $38.9 \%$ versus $31.7 \%$ for middle-low, $29.2 \%$ versus $30.2 \%$ for middle-high, and $13.9 \%$ versus $19.7 \%$ for highest SES status, respectively. About $50 \%$ in each group were married. Most of the patients in BCS (43.1\%) and mastectomy (42.5\%) groups had private insurance, $23.6 \%$ and $24.4 \%$ had Medicare, $5.6 \%$ and $10.2 \%$ had Medicaid, and $9.7 \%$ and $11.3 \%$ were uninsured, respectively. Few patients in each group had defense/military/veteran insurance and the rest had either unspecified or unknown insurance. More than half (63.9\%) of the patients with BCS have never smoked, $18.1 \%$ had past history of smoking, $9.7 \%$ were current smokers, compared to $58 \%, 16.7 \%$ and $14.2 \%$ of the women with mastectomy, respectively. Hospital volume was equally distributed between the two categories $(55.6 \%$ of BCS and $55.2 \%$ of mastectomy patients were treated in low volume), and the majority of the hospitals in each group (84.7\% in BCS and $79.1 \%$ in mastectomy) were non-teaching hospitals. Almost all the patients in our sample (692; 97.2\%) were from urban areas.

The clinical and histo-pathological characteristics of the patients are shown in table 2. The tumors of the BCS patients were positive for ER in $54.2 \%$ and for PR in $41.7 \%$ of patients compared to $48.3 \%$ and $37.8 \%$, respectively, in the mastectomy patients. Receptor status was unknown in $16.6 \%$ of the sample for ER and in $18 \%$ for the PR. Invasive ductal carcinoma dominated the histological type in both groups $86.1 \%$ and $79.8 \%$, followed by invasive lobular carcinoma in $6.9 \%$ and $13.4 \%$ in BCS and mastectomy, respectively. Our sample composed of only stage T3 and T4 breast cancers. Significantly more (64\%) of patient with BCS had T3 tumors, and more of mastectomy patients (59.5\%) had T4 disease ( $p<0.001)$. Comparing BCS versus mastectomy using the SEER stage at diagnosis, BCS group had significantly more localized disease $(23.6 \%)$, and less regional disease with direct extension and 


\begin{tabular}{|c|c|c|c|c|c|c|c|}
\hline \multirow{3}{*}{ Sociodemographic Characteristics } & \multirow{2}{*}{\multicolumn{2}{|c|}{ All Patients }} & \multicolumn{4}{|c|}{ Surgery } & \multirow{3}{*}{ p-value } \\
\hline & & & \multicolumn{2}{|c|}{ BCS } & \multicolumn{2}{|c|}{ Mastectomy } & \\
\hline & $\mathbf{n}$ & $\operatorname{col} \%$ & $\mathbf{n}$ & col\% & $\mathbf{n}$ & $\operatorname{col} \%$ & \\
\hline All & 712 & 100.0 & 72 & 10.1 & 640 & 89.9 & \\
\hline Status & & & & & & & 0.3034 \\
\hline Dead & 226 & 31.7 & 19 & 26.4 & 207 & 32.3 & \\
\hline Alive & 486 & 68.3 & 53 & 73.6 & 433 & 67.7 & \\
\hline \multicolumn{8}{|l|}{ Age } \\
\hline Mean (Sd) & \multicolumn{2}{|c|}{$55.4(13.7)$} & \multicolumn{2}{|c|}{$55.5(14.6)$} & \multicolumn{2}{|c|}{$55.4(13.6)$} & \\
\hline Median (Q1; Q3) & \multicolumn{2}{|c|}{$54(46 ; 64)$} & \multicolumn{2}{|c|}{$54(45 ; 62.5)$} & \multicolumn{2}{|c|}{$54(46 ; 64)$} & \\
\hline Min; $\max$ & \multicolumn{2}{|c|}{$22 ; 95$} & \multicolumn{2}{|c|}{$29 ; 90$} & \multicolumn{2}{|c|}{$22 ; 95$} & \\
\hline Race & & & & & & & 0.0975 \\
\hline White & 546 & 76.7 & 49 & 68.1 & 497 & 77.7 & \\
\hline Black & 153 & 21.5 & 20 & 27.8 & 133 & 20.8 & \\
\hline Other & 13 & 1.8 & 3 & 4.2 & 10 & 1.6 & \\
\hline Hispanic Origin & & & & & & & 0.1042 \\
\hline No & 619 & 86.9 & 67 & 93.1 & 552 & 86.3 & \\
\hline Yes & 93 & 13.1 & 5 & 6.9 & 88 & 13.8 & \\
\hline SES & & & & & & & 0.5337 \\
\hline Lowest & 131 & 18.4 & 13 & 18.1 & 118 & 18.4 & \\
\hline Middle-low & 231 & 32.4 & 28 & 38.9 & 203 & 31.7 & \\
\hline Middle-high & 214 & 30.1 & 21 & 29.2 & 193 & 30.2 & \\
\hline Highest & 136 & 19.1 & 10 & 13.9 & 126 & 19.7 & \\
\hline Marital Status & & & & & & & 0.3766 \\
\hline Unknown & 13 & 1.8 & 1 & 1.4 & 12 & 1.9 & \\
\hline Never Married & 149 & 20.9 & 19 & 26.4 & 130 & 20.3 & \\
\hline Married & 354 & 49.7 & 36 & 50.0 & 318 & 49.7 & \\
\hline Divorced/Separated/Widowed & 196 & 27.5 & 16 & 22.2 & 180 & 28.1 & \\
\hline Insurance Status & & & & & & & 0.3701 \\
\hline Unknown & 11 & 1.5 & 1 & 1.4 & 10 & 1.6 & \\
\hline Uninsured & 79 & 11.1 & 7 & 9.7 & 72 & 11.3 & \\
\hline Private Insurance & 303 & 42.6 & 31 & 43.1 & 272 & 42.5 & \\
\hline Medicaid & 69 & 9.7 & 4 & 5.6 & 65 & 10.2 & \\
\hline Medicare & 173 & 24.3 & 17 & 23.6 & 156 & 24.4 & \\
\hline Defense/Military/Veteran & 14 & 2.0 & 1 & 1.4 & 13 & 2.0 & \\
\hline Indian/Public & 3 & 0.4 & & & 3 & 0.5 & \\
\hline Insurance, NOS & 60 & 8.4 & 11 & 15.3 & 49 & 7.7 & \\
\hline \multirow[t]{3}{*}{ Sociodemographic Characteristics } & $\begin{array}{l}\text { All Pa- } \\
\text { tients }\end{array}$ & & & \multicolumn{3}{|c|}{ Surgery } & p-value \\
\hline & & \multicolumn{2}{|c|}{ BCS } & \multicolumn{2}{|c|}{ Mastectomy } & & \\
\hline & $\mathbf{n}$ & $\operatorname{col} \%$ & $\mathbf{n}$ & $\operatorname{col} \%$ & $\mathbf{n}$ & $\operatorname{col} \%$ & \\
\hline Tobacco Use & & & & & & & 0.6014 \\
\hline Never & 417 & 58.6 & 46 & 63.9 & 371 & 58.0 & \\
\hline History & 120 & 16.9 & 13 & 18.1 & 107 & 16.7 & \\
\hline Current & 98 & 13.8 & 7 & 9.7 & 91 & 14.2 & \\
\hline Unknown & 77 & 10.8 & 6 & 8.3 & 71 & 11.1 & \\
\hline Hospital Volume & & & & & & & 0.9485 \\
\hline Low & 393 & 55.2 & 40 & 55.6 & 353 & 55.2 & \\
\hline High & 319 & 44.8 & 32 & 44.4 & 287 & 44.8 & \\
\hline
\end{tabular}




\begin{tabular}{|c|c|c|c|c|c|c|c|}
\hline Teaching Hospital & & & & & & & \\
\hline No & 567 & 79.6 & 61 & 84.7 & 506 & 79.1 & 134 \\
\hline Yes & 145 & 20.4 & 11 & 15.3 & 20.9 & \\
\hline Residency & & & & & & \\
\hline Rural & 20 & 2.8 & 1 & 1.4 & 19 & 3.0 & \\
\hline Urban & 692 & 97.2 & 71 & 98.6 & 621 & 97.0 & \\
\hline
\end{tabular}

Table 1: Sociodemographic characteristics of female breast cancer patients by surgery type after PST.

\begin{tabular}{|c|c|c|c|c|c|c|c|}
\hline \multirow{3}{*}{ Clinical and histo-pathological Characteristics } & \multirow{2}{*}{\multicolumn{2}{|c|}{ All Patients }} & \multicolumn{4}{|c|}{ Surgery } & \multirow{3}{*}{ p-value } \\
\hline & & & \multicolumn{2}{|c|}{ BCS } & \multicolumn{2}{|c|}{ Mastectomy } & \\
\hline & $\mathbf{n}$ & $\operatorname{col} \%$ & $\mathbf{n}$ & col\% & $\mathbf{n}$ & $\operatorname{col} \%$ & \\
\hline All & 712 & 100.0 & 72 & 10.1 & 640 & 89.9 & \\
\hline Estrogen Receptor (ER) Assay & & & & & & & 0.3865 \\
\hline Unknown & 118 & 16.6 & 8 & 11.1 & 110 & 17.2 & \\
\hline Positive & 348 & 48.9 & 39 & 54.2 & 309 & 48.3 & \\
\hline Negative & 246 & 34.6 & 25 & 34.7 & 221 & 34.5 & \\
\hline Progesterone Receptor (PR) Assay & & & & & & & 0.4355 \\
\hline Unknown & 128 & 18.0 & 9 & 12.5 & 119 & 18.6 & \\
\hline Positive & 272 & 38.2 & 30 & 41.7 & 242 & 37.8 & \\
\hline Negative & 312 & 43.8 & 33 & 45.8 & 279 & 43.6 & \\
\hline Histology & & & & & & & 0.2929 \\
\hline Ductal Carcinoma & 573 & 80.5 & 62 & 86.1 & 511 & 79.8 & \\
\hline Lobular Carcinoma & 91 & 12.8 & 5 & 6.9 & 86 & 13.4 & \\
\hline Other & 48 & 6.7 & 5 & 6.9 & 43 & 6.7 & \\
\hline T Stage & & & & & & & $<0.001$ \\
\hline III & 305 & 42.8 & 46 & 63.9 & 259 & 40.5 & \\
\hline IV & 407 & 57.2 & 26 & 36.1 & 381 & 59.5 & \\
\hline SEER Summary Stage 2000 & & & & & & & $<0.0001$ \\
\hline Localized & 64 & 9.0 & 17 & 23.6 & 47 & 7.3 & \\
\hline Regional, direct extension & 93 & 13.1 & 12 & 16.7 & 81 & 12.7 & \\
\hline Regional, lymph nodes only & 167 & 23.5 & 16 & 22.2 & 151 & 23.6 & \\
\hline Regional, extension and nodes & 287 & 40.3 & 19 & 26.4 & 268 & 41.9 & \\
\hline Distant & 101 & 14.2 & 8 & 11.1 & 93 & 14.5 & \\
\hline Grade & & & & & & & 0.4517 \\
\hline Unknown/not stated & 108 & 15.2 & 9 & 12.5 & 99 & 15.5 & \\
\hline Well-differentiated & 29 & 4.1 & 1 & 1.4 & 28 & 4.4 & \\
\hline Moderately-differentiated & 167 & 23.5 & 18 & 25.0 & 149 & 23.3 & \\
\hline Poorly-differentiated/Undifferentiated & 408 & 57.3 & 44 & 61.1 & 364 & 56.9 & \\
\hline Regional Nodes Positive & & & & & & & 0.0027 \\
\hline Unknown & 91 & 12.8 & 13 & 18.1 & 78 & 12.2 & \\
\hline No & 162 & 22.8 & 25 & 34.7 & 137 & 21.4 & \\
\hline Yes & 459 & 64.5 & 34 & 47.2 & 425 & 66.4 & \\
\hline Number of Co-morbidity & & & & & & & 0.2791 \\
\hline None & 40 & 5.6 & 4 & 5.6 & 36 & 5.6 & \\
\hline $1 \sim 2$ & 69 & 9.7 & 10 & 13.9 & 59 & 9.2 & \\
\hline $3 \sim 4$ & 133 & 18.7 & 14 & 19.4 & 119 & 18.6 & \\
\hline$>4$ & 470 & 66.0 & 44 & 61.1 & 426 & 66.6 & \\
\hline
\end{tabular}

Table 2: Clinical and histo-pathological characteristics of female breast cancer patients by surgery type after PST. 
lymph node involvement (26.4\%), compared to mastectomy group (7.3\%) and (41.9\%), respectively. Regional disease with direct extension was $16.7 \%$ versus $12.7 \%$, regional with lymph nodes only was $22.2 \%$ versus $23.6 \%$, and distant metastatic disease was $11.1 \%$ versus $14.5 \%$ in BCS and mastectomy groups, respectively. The nuclear grade was comparable between the two groups, and more than half were poorly differentiated. We were missing the data for the status of the lymph nodes involvement in $12.8 \%$ of patients. For the patients with a known lymph nodes status, $47.2 \%$ had metastasis to lymph nodes in BCS group compared to $66.4 \%$ in mastectomy group, and $34.7 \%$ had no lymph node involvement in the BCS group compared to $21.4 \%$ in mastectomy group. More than half of the patients in BCS group (61.1\%) and mastectomy group (66.6\%) had more than four co-morbidities, and the number of co-morbidities was comparable between the two populations.
The type of PST provided was significantly different between the two groups ( $p=0.007)$. More of BCS patients $(12.5 \%)$ received preoperative hormonal therapy compared to only $4.4 \%$ of mastectomy patients. Preoperative chemotherapy was provided to $66.7 \%$ of BCS patients and $71.7 \%$ of mastectomy patients. Although combining chemotherapy with hormonal therapy is not recommended for the treatment of breast cancer, as the hormonal therapy can antagonize the effect of chemotherapy on the tumor by reducing the proliferation rate, our data shows that combined chemo/hormonal therapy were administered to $11.1 \%$ of BCS patients versus $16.7 \%$ of mastectomy patients. The timing and order of administration of each were not provided by the data, but we could assume that they were not given simultaneously. Radiation therapy was provided to $29.2 \%$ of BCS patients and to $33 \%$ of mastectomy patients (Table $3)$.

\begin{tabular}{|c|c|c|c|c|c|c|c|}
\hline \multirow{3}{*}{ Treatment Characteristics } & \multirow{2}{*}{\multicolumn{2}{|c|}{ All Patients }} & \multicolumn{4}{|c|}{ Surgery } & \multirow{3}{*}{ p-value } \\
\hline & & & \multicolumn{2}{|c|}{ BCS } & \multicolumn{2}{|c|}{ Mastectomy } & \\
\hline & $\mathbf{n}$ & col \% & $\mathbf{n}$ & col\% & $\mathbf{n}$ & col\% & \\
\hline All & 712 & 100.0 & 72 & 10.1 & 640 & 89.9 & \\
\hline \multicolumn{8}{|l|}{ Radiotherapy } \\
\hline Unknown & 31 & 4.4 & 3 & 4.2 & 28 & 4.4 & \\
\hline No & 449 & 63.1 & 48 & 66.7 & 401 & 62.7 & 0.5018 \\
\hline Yes & 232 & 32.6 & 21 & 29.2 & 211 & 33.0 & \\
\hline \multicolumn{8}{|l|}{ Neoadjuvant Therapy } \\
\hline Unknown & 53 & 7.4 & 7 & 9.7 & 46 & 7.2 & \\
\hline Chemotherapy & 507 & 71.2 & 48 & 66.7 & 459 & 71.7 & 0.0071 \\
\hline Hormonal Therapy & 37 & 5.2 & 9 & 12.5 & 28 & 4.4 & \\
\hline Chemo/Hormonal Therapy & 115 & 16.2 & 8 & 11.1 & 107 & 16.7 & \\
\hline
\end{tabular}

Table 3: Treatment characteristics of female breast cancer patients by surgery type after PST.

Table 4 shows the survival rates for the patients in each group. The one, three, and five- year survival rates for BCS patients was $91.1 \%, 68 \%, 55 \%$, and for mastectomy patients was $94.2 \%, 66.6 \%$, and $50 \%$, respectively.

Table 5 shows the results from a multivariate Cox regression model for overall survival. After adjusting for sociodemographic, tumor and treatment related characteristics and comorbidities. Survival between patients with mastectomy vs. BCS did not differ significantly (aHR $=1.35,95 \% \mathrm{CI}: 0.82-2.21, \mathrm{p}=0.234$ ). Patients who received both chemotherapy and hormonal preoperative therapy had better prognosis than patients with chemotherapy alone $(1.90,1.20-3.01, p=0.006)$, but not hormonal therapy alone (1.95, $0.84-4.56, \mathrm{p}=0.122$ ). Hispanic origin was associated with better survival $(0.50,0.27-0.92, p=0.027)$, compared to non-Hispanic. Compared to private insurance, uninsured patients had worse prognosis $(1.69,1.10-2.60, \mathrm{p}=0.016)$, and Medicare had 70\% more risk for worse survival $(1.70,1.08-2.68, p=0.021)$.

\begin{tabular}{|c|c|c|c|}
\hline Time & All \% (Range) & BCS \% (Range) & Mastectomy \% (Range) \\
\hline Median Survival & $5.2(4.4---7.0)$ & NA $(3.4-- \text { NA })^{*}$ & $5.2(4.4--7.0)$ \\
\hline 1 Year & $93.9 \%(91.8--95.4)$ & $91.1 \%(81.3--95.9)$ & $94.2 \%(92.0-95.8)$ \\
\hline 3 Years & $66.7 \%(62.5--70.6)$ & $68.0 \%(52.4--79.5)$ & $66.6 \%(62.2--70.6)$ \\
\hline 5 Years & $50.5 \%(44.6--56.1)$ & $55.0 \%(36.3-70.3)$ & $50.0 \%(43.8-55.9)$ \\
\hline
\end{tabular}

Table 4: Survival rate of female breast cancer patients by type of surgery.

* NA: Not attained. 


\begin{tabular}{|c|c|c|c|}
\hline Prognostic factors & Category & HR (95\% CI) & p-value \\
\hline \multirow{2}{*}{ Surgery } & BCS & 1.00 & \\
\hline & Mastectomy & $1.35(0.82,2.21)$ & 0.234 \\
\hline \multirow[t]{2}{*}{ Radiotherapy } & No & 1.00 & \\
\hline & Yes & $0.82(0.58,1.17)$ & 0.273 \\
\hline \multirow[t]{3}{*}{ PST } & Chemo/Hormonal Therapy & 1.00 & \\
\hline & Chemotherapy only & $1.90(1.20,3.01)$ & 0.006 \\
\hline & Hormonal Therapy only & $1.95(0.84,4.56)$ & 0.122 \\
\hline \multirow[t]{2}{*}{ Hispanic Origin } & No & 1.00 & \\
\hline & Yes & $0.50(0.27,0.92)$ & 0.027 \\
\hline \multirow[t]{8}{*}{ Insurance Status } & Private Insurance & 1.00 & \\
\hline & Defense/Military/Veteran & $1.17(0.45,3.04)$ & 0.740 \\
\hline & Indian/Public & $2.74(0.73,10.31)$ & 0.137 \\
\hline & Insurance, NOS & $1.24(0.65,2.36)$ & 0.509 \\
\hline & Medicaid & $1.53(0.82,2.88)$ & 0.184 \\
\hline & Medicare & $1.70(1.08,2.68)$ & 0.021 \\
\hline & Uninsured & $1.69(1.10,2.60)$ & 0.016 \\
\hline & Unknown & $2.16(0.83,5.60)$ & 0.114 \\
\hline \multirow[t]{2}{*}{ Residency } & Urban & 1.00 & \\
\hline & Rural & $0.50(0.26,0.97)$ & 0.040 \\
\hline \multirow[t]{4}{*}{ Grade } & Well-differentiated & 1.00 & \\
\hline & Moderately-differentiated & $1.21(0.51,2.87)$ & 0.669 \\
\hline & Poorly-differentiated/Undifferentiated & $2.24(1.04,4.82)$ & 0.039 \\
\hline & Unknown/not stated & $1.57(0.63,3.91)$ & 0.334 \\
\hline \multirow[t]{3}{*}{ Regional Nodes Positive } & None & 1.00 & \\
\hline & Yes & $1.82(0.95,3.48)$ & 0.072 \\
\hline & Unknown & $2.04(1.07,3.90)$ & 0.031 \\
\hline \multirow[t]{5}{*}{ SEER Summary Stage 2000} & Localized & 1.00 & \\
\hline & Distant & $4.13(1.68,10.12)$ & 0.002 \\
\hline & Regional, direct extension & $1.31(0.54,3.20)$ & 0.551 \\
\hline & Regional, extension and nodes & $2.08(0.89,4.90)$ & 0.092 \\
\hline & Regional, lymph nodes only & $1.25(0.51,3.08)$ & 0.627 \\
\hline \multirow[t]{3}{*}{ Histology } & Ductal Carcinoma & 1.00 & \\
\hline & Lobular Carcinoma & $1.05(0.65,1.70)$ & 0.847 \\
\hline & Other & $1.08(0.68,1.74)$ & 0.736 \\
\hline
\end{tabular}

Table 5: A multivariate cox regression models for overall survival $(n=630$ after excluded patients with unknown radiation therapy and chemo/hormonal therapy).

The following variables are also included in the model for adjustment but the results are not shown; race (White, Black, Other), SES (lowest, middle-low, middle-high, highest), age at diagnosis, tobacco use, hospital volume (high vs. low, teaching hospital (yes vs no).

Poorly-differentiated/undifferentiated tumors had significantly worse survival $(2.24,1.04-4.82, \mathrm{p}=0.039)$ compared to welldifferentiated ones, and distant SEER stage disease had significantly very poor prognosis $(4.13,1.68-10.12, \mathrm{p}=0.002)$ compared to localized disease.

The administration of radiotherapy, nodal status, and histological type of tumor did not significantly impact survival between the two groups in a multivariate analysis (Table 5), neither did the race, SES, age at diagnosis, marital status, teaching/ nonteaching hospital, nor hospital volume (data not shown). However, in a univariate analysis of overall survival in patients who underwent mastectomy, radiotherapy was associated with a statistically significant better overall survival (HR 0.68, $0.47-0.99, \mathrm{p}=0.044$ ).

Kaplan-Meier survival curves were depicted as shown in figure 1 , demonstrating in figure $1 \mathrm{~A}$ no statistical difference $(\mathrm{p}=0.774$ ) 

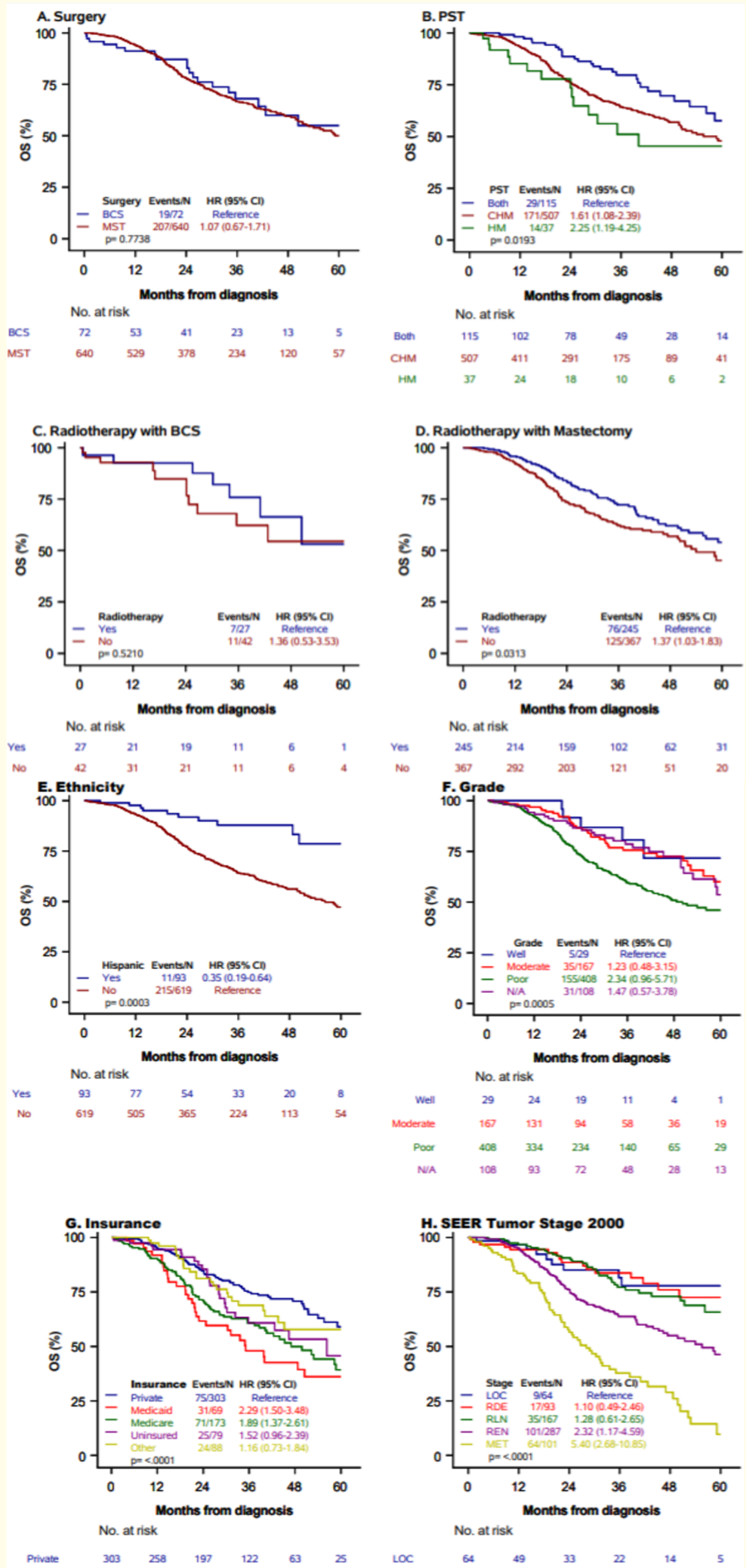

Figure 1: Kaplan Meier survival curves; A. by type of surgery, B. by type of PST, C. by radiotherapy with BCS, D. by radiotherapy with mastectomy, E. by ethnicity, F. by tumor grade, G. by insurance, H. by SEER tumor stage; LOC: Localized; RDE: Regional, direct extension; RLN: Regional, lymph nodes only; REN: Regional, extension and nodes; MET: Distant metastasis. 
in survival based on the type of surgery (BCS or mastectomy). Combination preoperative chemo/hormonal therapy resulted in significantly better survival ( $p=0.019)$, compared to any of these modalities alone (Figure 1B). Within a subgroup of patients in each type of surgery, radiation therapy did not significantly $(\mathrm{p}=0.521)$ impact survival for BCS patients (Figure 1C). However, it was associated with better survival $(p=0.031)$ for mastectomy patients (Figure 1D).

Survival was better $(p=0.0003)$ for Hispanic ethnicity (Figure $1 E)$, while poorly differentiated tumors ( $p=0.0005)$, lack of insurance $(\mathrm{p}<0.0001)$, and distant metastatic SEER stage $(\mathrm{p}<0.0001)$ were associated with worse survival (Figure $1 \mathrm{~F}-1 \mathrm{H}$ ) respectively. Although urban residential area was significant for better survival on multivariate analysis, it was not significant ( $p=0.971$ ) on univariate analysis, data not shown.

\section{Discussion}

Our study shows that type of surgery after PST does not impact survival. This was thought to be only true in early stage breast cancer; a recent study with 20 years follow up showed no difference in survival after BCS or mastectomy for stage I-II breast cancer [4]. In the recent NSABP-B18 trial comparing preoperative versus postoperative chemotherapy in women with resectable carcinoma of the breast, the local recurrence rates were significantly higher in patients with large tumors who underwent lumpectomy after neoadjuvant chemotherapy [5]. However, the study was not initially conducted to compare between mastectomy and lumpectomy. On the other hand, a study that reported the long-term outcome of neoadjuvant therapy for locally advanced breast cancer showed that survival after BCS was better than after mastectomy $(90 \%$ vs $50 \%$ ) [6]. And in a French study, the five- and ten-year overall survival rates and disease-free survival rates, after neoadjuvant therapy and BCS, were $80 \%, 69 \%, 73 \%$, and $61 \%$ respectively [7]. Fitzal and colleagues also concluded that BCS itself was not an independent prognostic factor for a worse loco-regional disease free survival, and overall survival was better for BCS group, compared to mastectomy group [8]. These data indicate that after PST, BCS is equivalent to mastectomy in terms of patient survival, even for large tumors. This is provided the receipt of post lumpectomy radiotherapy and is greatly influenced by the tumor response to PST [6].

This paper shows that patients of Hispanic origin had better survival than patients of non-Hispanic origin. This could be surprising because the data in the literature show that Hispanic patients present with a more aggressive tumor profile associated with worse prognosis, compared to non-Hispanic patients [9-11]. And hence, one would expect Hispanic patients to have worse survival. Indeed, Shavers and colleagues have demonstrated in their paper that African American and Hispanic women with breast cancer had poorer overall survival compared to White women [12]. The finding in our study could be explained by the low number of Hispanic patient in the sample (13\%) which might have skewed this result. This could also be explained with the lower incidence of breast cancer in Hispanic women to start with. The incidence of breast cancer is highest in White American, followed by African American, Asian American, and Hispanic women [13]. It should be mentioned however; that what we observed in our study is a well described phenomenon in the literature called "Hispanic paradox" in which most Hispanic groups are characterized by low SES, but better than expected health and mortality outcomes. Although the true explanation to this phenomenon is not well understood, some suggest that under-reporting of Hispanic deaths and healthy migrant effects are possible explanations [14], however, no data to support that.

In contrast to other studies, our study also shows that residents of rural areas have better survival compared to residents in urban areas. This finding, again, is likely due to the very small number of events (7 events) in rural residency, which is also compromising only $2.9 \%$ of the population sample. A study in Poland showed less overall survival for patients residing in rural areas [15]. Another study in Australia showed that the five-year survival for breast cancer patients was significantly lowered in rural, compared to urban areas [16]. This is an expected outcome of limited access to medical care and inadequate comprehensive diagnosis and treatment provided in rural areas [17].

The type of insurance was a significant predictor of survival in our sample, with uninsured and Medicare patients having the worst prognosis compared to private insurance patients. Two recent studies covering the same period of time have similarly found that uninsured patients have poor survival compared to insured patients. However, Medicare was not associated with lower rates of survival in those studies $[18,19]$. A possible explanation is a discrepancy in the level of service provided by Medicare among different states in the country. While the former mentioned studies looked into National Cancer Database for patients from all over the country, our study only looked at Florida population based database. This raises a question about the quality of health insurance provided by Medicare to Florida residents.

Radiotherapy seemed to improve survival after PST and mastectomy in our study. This is consistent with current data in the literature. Huang and colleagues analyzed six consecutive institutional prospective trials with neoadjuvant chemotherapy, mastectomy, and radiation [20]. They concluded that after neoadjuvant therapy and mastectomy, radiation has significantly reduced loco-regional recurrence and improved cause-specific survival in patients presenting with clinical T3 tumors or stage III-IV (ipsilateral supraclavicular nodal) disease. On the other hand, radiation for BCS patients was not a significant predictor of survival in our study. Although radiotherapy is the standard of care after BCS [21], its main role is prevention of local recurrence and only a much smaller improvement in survival. This small survival benefit was only apparent in 
large meta-analyses as the Early Breast Cancer Trialists Collaborative Group [22]. In the case of BCS after PST it is reasonable to assume that patients offered BCS were those with better response to treatment. The fact that neoadjuvant therapy can achieve complete pathological response (PCR) in the primary tumor as well as the lymph nodes, renders the benefit of adjuvant radiotherapy for loco-regional control questionable. A combined analysis of NSABP trials 18 and 27 has shown that the patients who had PCR after neoadjuvant therapy had the lower rates of loco-regional recurrence regardless of the type of surgery performed [23]. The authors suggested that it is possible to predict the patients who will need radiotherapy after neoadjuvant therapy and surgery based on their PCR. This very hypothesis is currently being studied in a large cooperative trial by NRG and results are pending [24]. We noticed that $73.6 \%$ of BCS patients are still alive although $66.7 \%$ of them did not receive adjuvant radiotherapy. This could be attributed to the effect of systemic therapy they received. The reason for not receiving adjuvant radiotherapy is unclear from the data. However, because adjuvant radiotherapy after BCS is the standard of care, we speculate that it was offered to the patients as part of the treatment plan, and we assume there were other reasons why those patients did not receive radiotherapy, such as living in rural area away from providing facility, lack of transportation, poor compliance or insurance limitation.

Our results show that poorly differentiated tumor grade is a predictor of poor survival after PST, irrespective to type of surgery. This is consistent with the finding from a previous study showing that low histological tumor grade is a favorable prognostic factor for survival after neoadjuvant therapy for operable disease [25]. Our database of PST received prior to the date of surgery included about $14 \%$ of patients with distant metastatic disease at diagnosis. As expected, these patients had significantly worse survival compared to patients with localized disease. We speculate that those patients received palliative systemic treatment and that surgery was performed for local control of the disease. Unfortunately, SEER data does not specify the purpose of systemic therapy whether it is for curative or palliative intent, and there is no specific code in the ICD-10 for "palliative" chemotherapy.

The type of PST was an independent predictor of survival is our data; the patients who received both preoperative chemo/ hormonal therapies had better survival compared to patients who received chemotherapy alone. This is probably because these patients had ER positive tumors and therefore, less aggressive and more favorable disease compared to ER negative tumors. As stated in the results section PST does not typically combine chemotherapy with hormonal therapy. Furthermore, for ER positive patients who are treated with neoadjuvant chemotherapy, the endocrine therapy is usually deferred till after the surgery. In our study, however, a subgroup of patients received both hormonal and chemotherapy in the neoadjuvant setting and this subgroup specifically had a better survival. It would be reasonable to assume that this group consists of ER positive patients who were treated initially with hormonal therapy then were switched to chemotherapy after no significant response was observed. Genomic profiling used sometimes in the neoadjuvant setting might help better select the type of neoadjuvant therapy. We did not find that patients who received preoperative hormonal therapy only had improved survival compared to other groups in our study. This finding may be explained by the low utilization of preoperative hormonal therapy in our population. While $49 \%$ of the patients had ER positive cancer, only $5 \%$ received preoperative hormonal therapy.

\section{Conclusion}

The type of breast surgery (BCS or mastectomy) after PST for T3/T4 breast cancer does not impact overall survival. BCS is still an option to be considered when planning surgical treatment after PST. Irrespective of type of surgery, tumor grade, type of PST, ethnicity, post mastectomy radiotherapy and health insurance are significant predictors of survival after PST. Serious measures should be implicated to adjust controllable factors and improve breast cancer survival in these patients.

This study was funded by James and Esther King Florida Biomedical Research Program (\#10KG-06; PI: Margaret M. Byrne). The authors confirm that the funder had no influence over the study design, content of the article, or selection of this journal.

All authors have no conflict of interest. The Florida cancer incidence data used in this report were collected by the Florida Cancer Data System (FCDS), the statewide cancer registry funded by the Florida Department of Health (DOH) and the Centers for Disease Control and Prevention's National Program of Cancer Registries (CDC-NPCR). The views expressed herein are solely those of the author(s) and does not necessarily reflect those of the DOH or CDCNPCR.

\section{Authors Contributions}

Jamila Al-Azhri ${ }^{1,3,4,5,6}$, Tulay Koru-Sengul ${ }^{1,2,3,4,5,6,7}$, Feng Miao ${ }^{2,3,4,5,6}$, Margaret M. Byrne ${ }^{2,3,4,5,6,7}$, Eli Avisar ${ }^{1.3,5,6}$

1. Substantial contributions to conception and design.

2. Acquisition of data and data analysis.

3. Interpretation of data.

4. Drafting the article or revising it critically for important intellectual content.

5. Final approval of the version to be published.

6. Agreement to be accountable for all aspects of the work.

7. Secure competitive funding for the study. 


\section{Bibliography}

1. J Al-Azhri., et al. "Predictors of surgery types after neoadjuvant therapy for advanced stage breast cancer: Analysis from Florida population-based cancer registry (1996-2009)". Breast Cancer (Auckl) 9 (2015): 99-108.

2. Hwang ES., et al. "Survival after lumpectomy and mastectomy for early stage invasive breast cancer". Cancer 119.7 (2013): 1402-1411.

3. Cho JH., et al. "Oncologic safety of breast-conserving surgery compared to mastectomy in patients receiving neoadjuvant chemotherapy for locally advanced breast cancer". Journal of Surgical Oncology 108.8 (2013): 531-536.

4. Litiere S., et al. "Breast conserving therapy versus mastectomy for stage I-II breast cancer: 20 year follow-up of the EORTC 10801 phase 3 randomised trial". Lancet Oncology 13.4 (2012): 412-419.

5. Fisher B., et al. "Effect of preoperative chemotherapy on local-regional disease in women with operable breast cancer: findings from National Surgical Adjuvant Breast and Bowel Project B-18". Journal of Clinical Oncology 15.7 (1997): 24832493.

6. Cance WG., et al. "Long-term outcome of neoadjuvant therapy for locally advanced breast carcinoma - Effective clinical downstaging allows breast preservation and predicts outstanding local control and survival". Annals of Surgery 236.3 (2002): 295-303.

7. Touboul E., et al. "Possibility of conservative local treatment after combined chemotherapy and preoperative irradiation for locally advanced noninflammatory breast cancer". International Journal of Radiation Oncology 34.5 (1996): 1019-1028.

8. Fitzal F., et al. "Oncologic safety of breast conserving surgery after tumour downsizing by neoadjuvant therapy: a retrospective single centre cohort study". Breast Cancer Research and Treatment 127.1 (2011): 121-128.

9. Martinez ME., et al. "Breast cancer among Hispanic and nonHispanic White women in Arizona". Journal of Health Care for the Poor and Underserved 18.4 (2007): 130-145.

10. Banegas MP and Li CI. "Breast cancer characteristics and outcomes among Hispanic Black and Hispanic White women". Breast Cancer Research and Treatment 134.3 (2012): 12971304.

11. Frost F., et al. "Breast cancer survival among new Mexico Hispanic, American Indian, and non-Hispanic white women (1973-1992)". Cancer Epidemiology, Biomarkers and Prevention 5.11 (1996): 861-866.
12. Shavers VL., et al. "Racial/ethnic variation in clinical presentation, treatment and survival among breast cancer patients under age 35". Cancer Epidemiology, Biomarkers and Prevention 11.10 (2002): 1203s-1203s.

13. Smigal C., et al. "Trends in breast cancer by race and ethnicity: Update 2006". CA: A Cancer Journal for Clinicians 56.3 (2006): 168-183.

14. Franzini L., et al. "Understanding the Hispanic paradox". Ethnicity and Disease. Autumn 11.3 (2006): 496-518.

15. Krzyzak M., et al. "Breast Cancer Survival Gap between Urban and Rural Female Population in Podlaskie Voivodship, Poland, in 2001-2002. Population Study". Annals of Agricultural and Environmental Medicine 17.2 (2010): 277-282.

16. Mitchell KJ., et al. "Rural-urban differences in the presentation, management and survival of breast cancer in Western Australia". Breast 15.6 (2006): 769-776.

17. Howe HL., et al. "Urban-Rural Differences in the Management of Breast-Cancer”. Cancer Cause Control 4.2 (1993): 172-172.

18. Shi RH., et al. "Effects of payer status on breast cancer survival: a retrospective study". BMC Cancer 15 (2015).

19. Shi RH., et al. "Disparities in breast cancer relative survival according to payer status: Findings from national cancer data bank". Cancer Research 74.19 (2014): 4128.

20. Huang EH., et al. "Postmastectomy radiation improves localregional control and survival for selected patients with locally advanced breast cancer treated with neoadjuvant chemotherapy and mastectomy". Journal of Clinical Oncology 22.23 (2004): 4691-4699.

21. Mak KS and Harris JR. "Radiotherapy Issues After Neoadjuvant Chemotherapy". Journal of the National Cancer Institute. Monographs 2015.51 (2015): 87-89.

22. Collab EBCT. "Effect of radiotherapy after breast-conserving surgery on 10-year recurrence and 15-year breast cancer death: meta-analysis of individual patient data for 10801 women in 17 randomised trials". Lancet 378.9804 (2011): 1707-1716.

23. Smith BD. "Using Chemotherapy Response to Personalize Choices Regarding Locoregional Therapy: A New Era in Breast Cancer Treatment?". Journal of Clinical Oncology 30.32 (2012): 3913-3915. 
24. Mamounas EP., et al. "NRG Oncology/NSABP B-51/RTOG 1304: Phase III trial to determine if chest wall and regional nodal radiotherapy (CWRNRT) post mastectomy or the addition of RNRT to whole breast RT post breast-conserving surgery reduces invasive breast cancer recurrence-free interval (IBCR-FI) in patients with pathologically positive axillary nodes who are ypN0 after neoadjuvant chemotherapy". Journal of Clinical Oncology 37 (2019): 15.

25. Pierga JY., et al. "Prognostic factors for survival after neoadjuvant chemotherapy in operable breast cancer: the role of clinical response". European Journal of Cancer 39.8 (2003): 1089-1096.

\section{Volume 5 Issue 6 June 2021}

(C) All rights are reserved by Jamila Alazhri., et al. 